\title{
Diversity Analysis among Chickpea Genetic Stock as Revealed Through STMS Marker Analysis
}

\author{
Nandakini Lahiri ${ }^{1}$, Tapan Kumar², C. Bharadwaj ${ }^{2 *}$, Ashutosh Sarker ${ }^{3}$, Aqeel Hasan Rizvi ${ }^{3}$, SK Chauhan ${ }^{2}$, \\ AK Verma² and Guru Prasad' \\ ${ }^{1}$ School of Life Sciences, Devi Ahilya Vishwavidyalaya, Indore-452001, Madhya Pradesh, India \\ ${ }^{2}$ Division of Genetics, ICAR-Indian Agricultural Research Institute, Pusa Campus, New Delhi-110012, India \\ ${ }^{3}$ ICARDA Office for South Asia, NASC Complex, New Delhi-110012, India
}

(Received: 25 July 2014; Revised: 24 December 2014; Accepted: 30 March 2015)

\begin{abstract}
Genetic diversity analysis of chickpea germplasm can provide useful information for the selection of parental material and thus help in planning breeding strategies.In the present study, a total of 57 STMS loci were analyzed to discern the variability among 87 chickpea lines consisting of released varieties and elite germplasm. A total of 87 alleles were found for the 19 STMS loci with an average of 4.57 alleles per locus. PIC value ranged from 0.94 to 0.10 and the heterozygosity ranged from 0.11 to 0.94 , indicating good variability among the material as well as polymorphism generated. All the genotypes could cluster into six distinct groups with one genotype remaining unclustered. Greater gains can be obtained by crossing lines MPJG-2000-108 with SBD 377 for Desi and PG 0515 with ILC 212 for Kabuli improvement. Base broadening through Kabuli $\times$ Desi introgression with greater gains can be obtained by using ICC 4516 and ILC 212 as parents.
\end{abstract}

Key Words: Chickpea, Cluster Analysis, Molecular markers, PCR, STMS markers

\section{Introduction}

Chickpea (Cicer arietinum L.; Family: Fabaceae) is a self-pollinated, diploid $(2 \mathrm{n}=16)$, cool season pulse crop with a genome size of $\sim 738-\mathrm{Mb}$ and an estimated 28,269 genes (Varshney et al., 2013). It is widely grown in more than 50 countries representing all the continents (Upadhyay et al., 2011). Worldwide chickpea ranks third among legumes (Food and Agricultural Organization, 2010) i.e. almost $15 \%$ of the total pulse production of world. In the duration of 2010, the worldwide chickpea area was about 12.0 million ha, with 10.9 million metric tons of production with the yield of $911 \mathrm{~kg} / \mathrm{ha}$ (FAOSTAT, 2012). India is the world's major producer, the annual production is around $7.58 \mathrm{Mt}$, grown in the area of approximately $8.32 \mathrm{mha}$, which is the world's $68 \%$ production of total chickpea and the average yield is approximately $912 \mathrm{~kg} / \mathrm{ha}$ (FAOSTAT, 2012). More than $95 \%$ of the area of production and consumption of chickpea is shared by the developing countries. Chickpea is grown mainly in South East Asian countries. Kabuli (white seeds) and Desi (brown seeds) are the two main types of cultivated chickpea, presenting two diverse gene pools (Nawroz and Hero, 2011).

\footnotetext{
*corresponding author: E-mail: chbharadwaj@yahoo.co.in
}

Chickpea has a very narrow genetic base which is limiting the genetic improvement of chickpea through breeding efforts. The level of natural variation among cultivated chickpea and wild accessions at molecular level is greatly aids in increasing the efficiency of breeding programme (Bharadwaj et al., 2011). This is because the phenotypic variability is largely an account of ' $\mathrm{G} \times \mathrm{E}$ ' interaction where as the variability at molecular level is devoid of the interference by environment. Diversity analysis is essential to understand per se the variability present in germplasm collection that can be practically put to use in plant breeding programmes for recombination breeding. Simple Sequence Repeats (SSRs) are the preferred markers in most areas of molecular genetics as they are highly polymorphic even between closely related lines require very low amount of DNA and are very transferable across populations. SSRs are generally co-dominant markers and are most useful for studies on population genetics and mapping (Jarne and Lagoda, 1996; Goldstein and Pollock, 1997). SSR genotypic data from a number of loci have potential to provide distinctive allelic profiles for establishing genotypes identity (Bharadwaj et al., 2010, 2011; Chaudhary et al., 2012). Keeping the above points in mind an 
investigation was planned to discern the variability of diverse chickpea lines consisting of elite germplasm and cultivated lines, so that most diverse parents for crossing programme can be identified and diversity of the material can be analyzed.

\section{Materials and methods}

A total of 87 elite chickpea genetic stock obtained from Chickpea Project, Division of Genetics, Indian Agricultural Research Institute, New Delhi 110012 were used in this study. The genotypes were designated as GS-1 to GS-87 (Table 1).

\section{DNA Isolation and Genotyping}

Isolation of DNA was carried out by as per Kumar et al., 2013. A total of 50 sequence tagged microsatellite site (STMS) loci were screened in the accessions of which only 19 were polymorphic (Table 2). The STMS markers were synthesized as per the sequences of (Winter et al., 2000; Bharadwaj et al., 2010) from Bioneer, Daejeon, South Korea. BioRad MyCycler thermal cycler, Richmond, USA was used to carry out amplifications in $10 \mu \mathrm{L}$ volume reaction mixture. This mixture contained $1 \mu \mathrm{L}$ of 20 ng plant genomic DNA, $1.6 \mu \mathrm{L}$ of $10 \times$ Tris buffer $(15 \mathrm{mM} \mathrm{MgCl} 2$ and gelatine), $1 \mu \mathrm{L}$ of $10 \mathrm{mM}$ dNTP mix, $1.0 \mu \mathrm{L}$ each of forward and reverse primer and $0.3 \mu \mathrm{L}$ of $3 \mathrm{U}_{\mu} \mathrm{L}^{-1} T a q$ polymerase. PCR was performed with following conditions $50 \mathrm{~s}$ at $90^{\circ} \mathrm{C}$ followedby 18 cycles of denaturation at $94^{\circ} \mathrm{C}$ for $20 \mathrm{~s}$, annealing for $50 \mathrm{~s}$ at $50^{\circ} \mathrm{C}$ (Touch down of $0.5^{\circ} \mathrm{C}$ for every repeat cycle) and 1 min elongation at $72^{\circ} \mathrm{C}$ for $50 \mathrm{~s}$. Further 20 cycles of denaturationat $94^{\circ} \mathrm{C}$ for $20 \mathrm{~s}$, annealing for $50 \mathrm{~s}$ at $55^{\circ} \mathrm{C}$ and $50 \mathrm{~s}$ elongation at $72^{\circ} \mathrm{C}$ were given and final extension at $72^{\circ} \mathrm{C}$ for 7 min were performed. The resolution of PCR products was done on three per cent metaphor gels (Lonza) (Fig. 1).

The polymorphic bands were scored in a spread sheet format with ' 0 ' representing absence of band and ' 1 ' representing the presence of band 'Null allele' for any specific marker in any genotype was again considered as absence of band (designated as ' 0 '). The data was analyzed in NTSYS-PC software (version 2.21b). Bootstraps were done using Free Tree and Tree view software. For Clustering, UPGMA was used based on the similarity matrix generated on combined data. Polymorphic information content for each STMS primer pair was calculated.

\section{Results}

In the present study, a total of 57 STMS loci were analyzed, covering various bin locations on different linkage groups of which 19 were polymorphic (Table 2). A lower level of polymorphism is expected in chickpea which is having a narrow level of diversity compared to other crops and here it was $33.3 \%$. All the 19 STMS loci, in the genetic material under study were found to be highly polymorphic. Excellent polymorphism was revealed by most of these STMS markers. Data from all the 19 STMS loci were utilized for statistical analysis. A total of 87 alleles were found with an average of 4.57 alleles per locus. The highest numbers of alleles were observed in TA194 (five alleles), TA 14, TA80, TA113, TA117 (four alleles each), TA14, TA71, TA110, CaSTMS2, CaSTMS15 and NCPGR4 (three alleles each).

Polymorphism information content (PIC) of each marker system was calculated for each marker and locus using the polymorphism information content (Lynch and Walsh, 1998) which gives an estimate of the discriminating power of a locus by taking into account not only the number of alleles that are expressed but also their relative frequencies. PIC ranged from 0.11 to 0.94 . Highest PIC was observed for NCPGR7 and lowest for TA71.

Some STMS markers were found to have high discriminative power for differentiation of chickpea genetic stocks as the present study demonstrates that 19 out of 57 STMS alleles were found to be unique or rare; unique or rare allele is one with a frequency less than or equal to 0.10 . The present findings also indicated instances where the STMS profiles for some of the genotypes displayed maximum variation pattern. Chickpea is highly self-pollinated and should, therefore, reveal lower polymorphism for majority of the genotypes, thus the occurrence of dialleles is also very less with a few primers only and is in concurrence with the reports (Singh et al., 2008; Ahmad et al., 2010; Singh et al., 2014) of a narrow genetic base of chickpea. It has been The STMS data was utilized for estimating pair wise genetic similarities among various entries using Jaccard's coefficient (1908) method. The genetic similarity matrix was further analyzed using UPGMA clustering algorithm by software programme NTSYS pc version $2.21 \mathrm{~b}$. The dendogram derived from this analysis is depicted in Fig. 2. The dendogram clearly showed 5 large clusters, 1 small clusters and 1 genotype remained ungrouped (GS39). The 


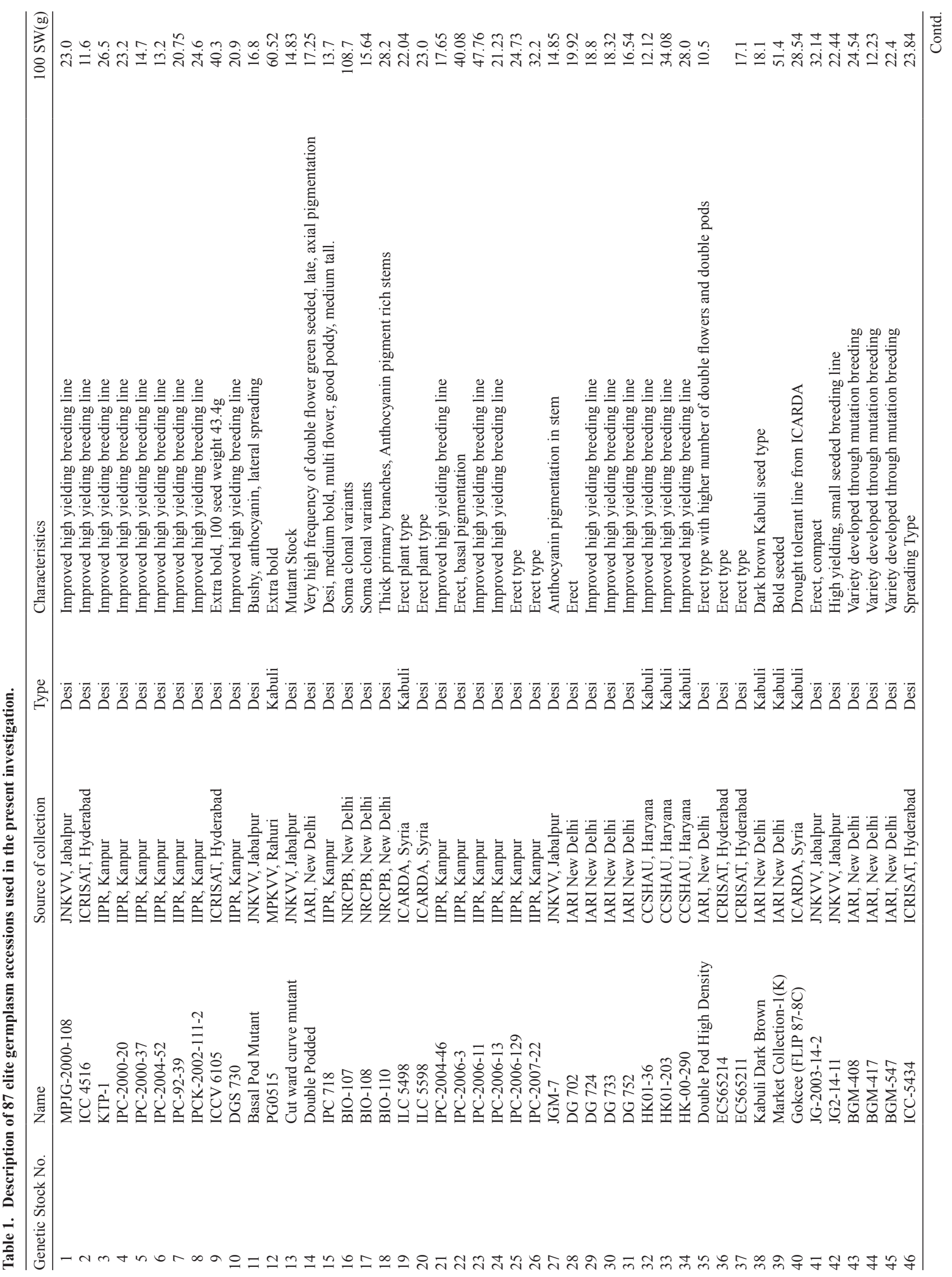




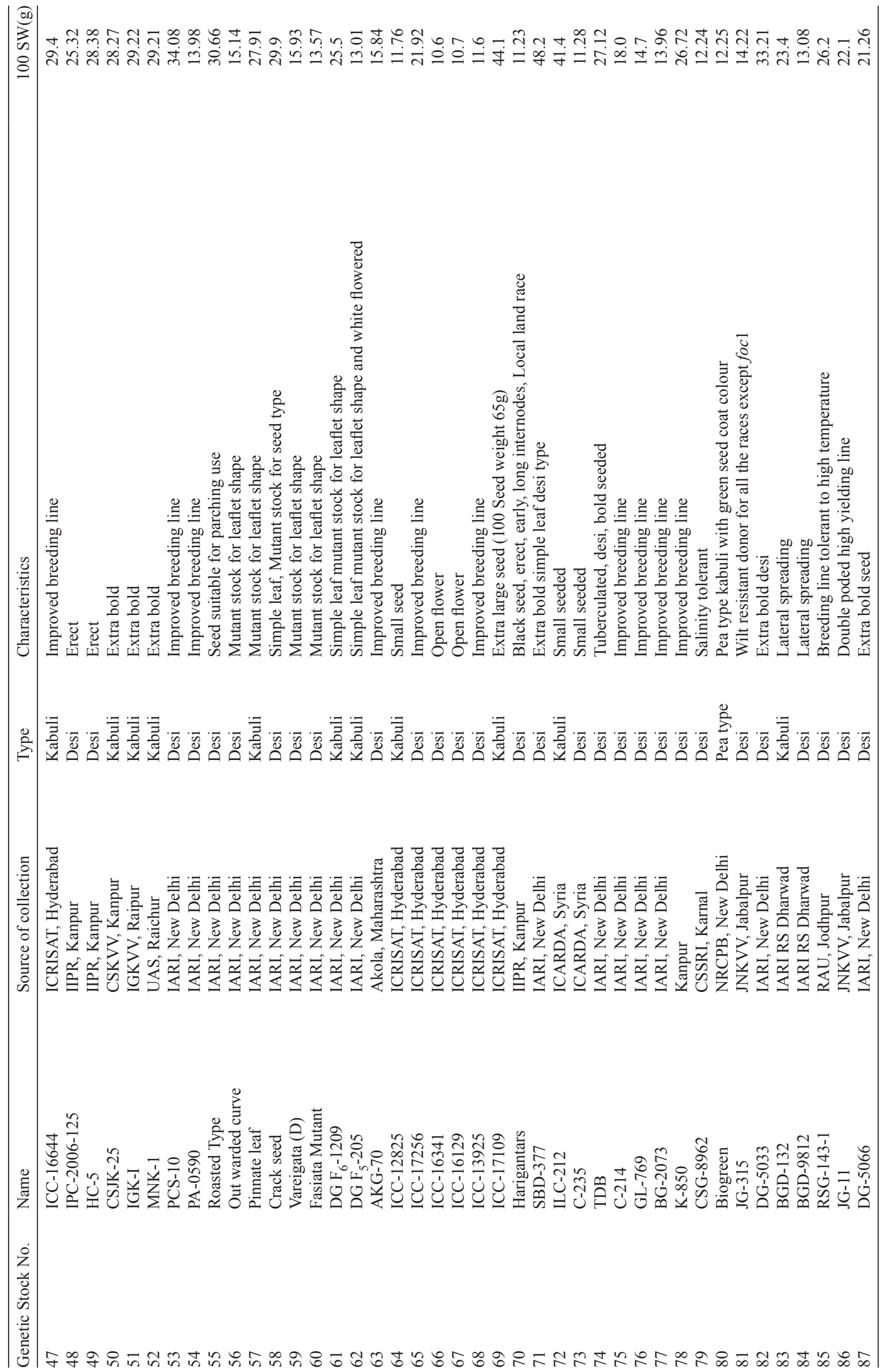




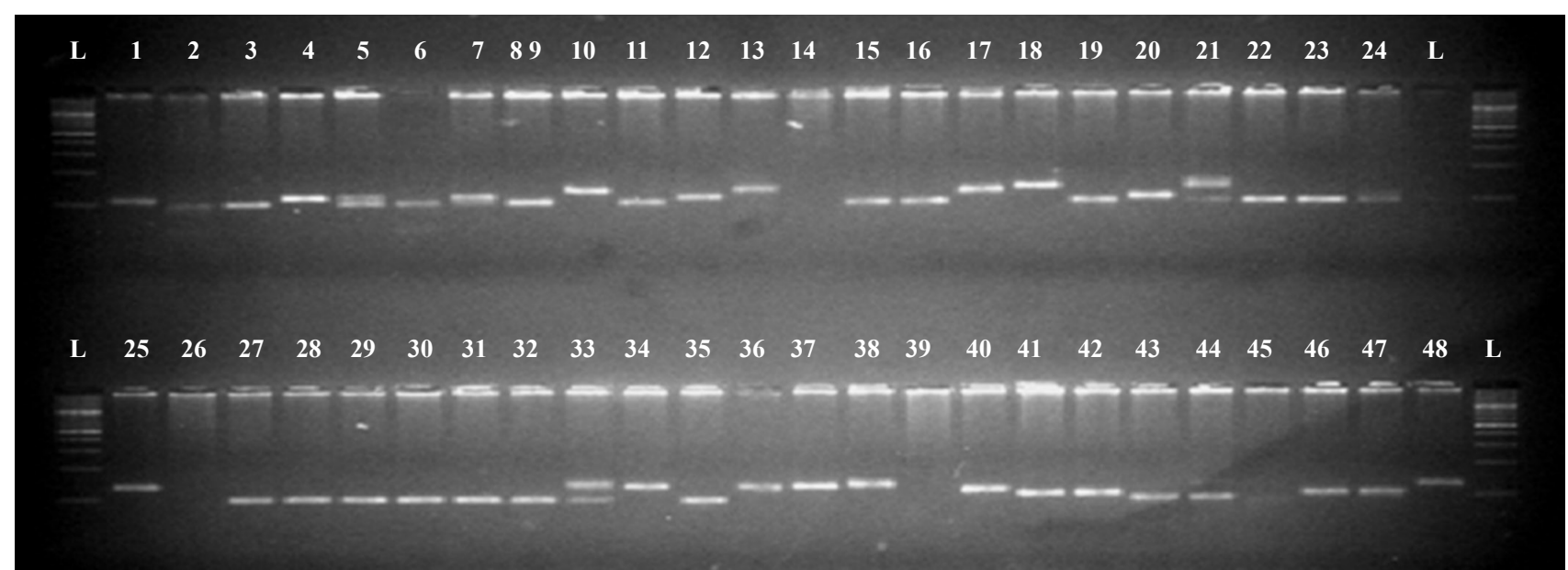

Fig. 1(a) SSR-PCR amplification products of chickpea with accessions using SSR primer TA194

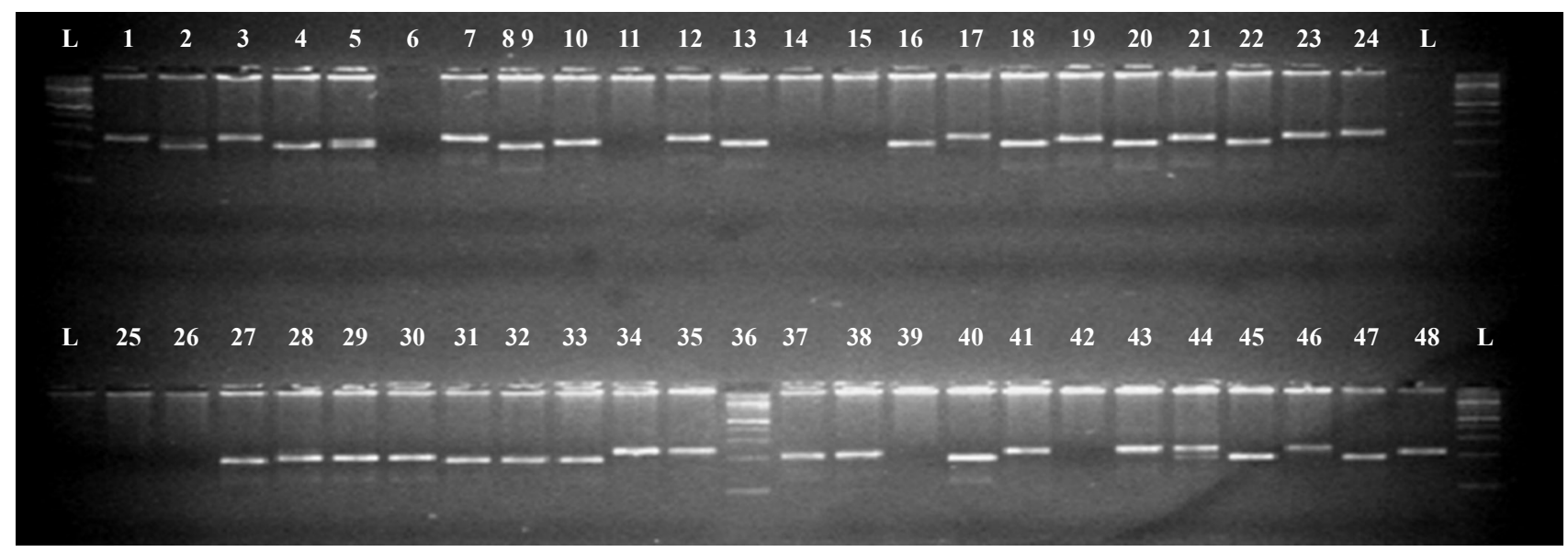

Fig. 1(b) SSR-PCR amplification products of chickpea with accessions using SSR primer TA80

Table 2. SSR primers used for chickpea germplasm analysis

\begin{tabular}{clccc}
\hline S. No. & Primer name & Linkage group & PIC value & Heterozygosity \\
\hline 1 & TA103 & LG2 & 0.33 & 0.42 \\
2 & TA3 & LG9 & 0.18 & 0.20 \\
3 & TA14 & LG4 & 0.47 & 0.53 \\
4 & TA71 & LG5 & 0.11 & 0.11 \\
5 & TA186 & LG4 & 0.21 & 0.23 \\
6 & TA194 & LG2 & 0.68 & 0.72 \\
7 & TA203 & LG1 & 0.17 & 0.17 \\
8 & TA200 & LG2 & 0.59 & 0.59 \\
9 & TA80 & LG6 & 0.59 & 0.66 \\
10 & TA96 & LG7 & 0.25 & 0.29 \\
11 & TA113 & LG 1 & 0.45 & 0.53 \\
12 & TA117 & LG2 & 0.47 & 0.56 \\
13 & TA110 & LG2 & 0.40 & 0.52 \\
14 & CaSTMS2 & a & 0.10 & 0.11 \\
15 & CaSTMS15 & a & 0.51 & 0.60 \\
16 & NCPGR4 & LG6 & 0.44 & 0.52 \\
17 & NCPGR6 & LG6 & 0.84 & 0.84 \\
18 & NCPGR7 & LG4 & 0.94 & 0.94 \\
19 & NCPGR12 & LG7 & 0.27 & 0.32 \\
\hline
\end{tabular}

Source: Bharadwaj et al., 2010, 2011; a - from Huttel et al.; Varshney et al., 2013.

Indian J. Plant Genet. Resour. 28(2): 189-197 (2015) cluster I, II, III, IV, V and VI comprised of 36, 5, 13, 11,19 and 2 genotypes respectively. Maximum Jaccard's correlation was seen for the genotypes IPC-2000-20 (GS4) and IPC-2000-37 (GS5) while the genotypes BGD-132 (GS83) and BGD-9812(GS84) were having highest similarity index, while the genotypes SBD377 (GS71) and ILC-212(GS72) have shown the highest dissimilarity with all the other genotypes and distinctly formed a separate cluster (cluster VI) (Fig. 2).

\section{Discussions}

Among the various DNA based markers, microsatellite or STMS markers are highly accepted and have been used in the diverse crop plants owing to their abundance in the genome (Powell et al., 1996). The application of STMS markers in genetic analysis of chickpea, started with an initial study of (Huttel et al., 1999) and after that, the power and potential of SSR markers for a broad 


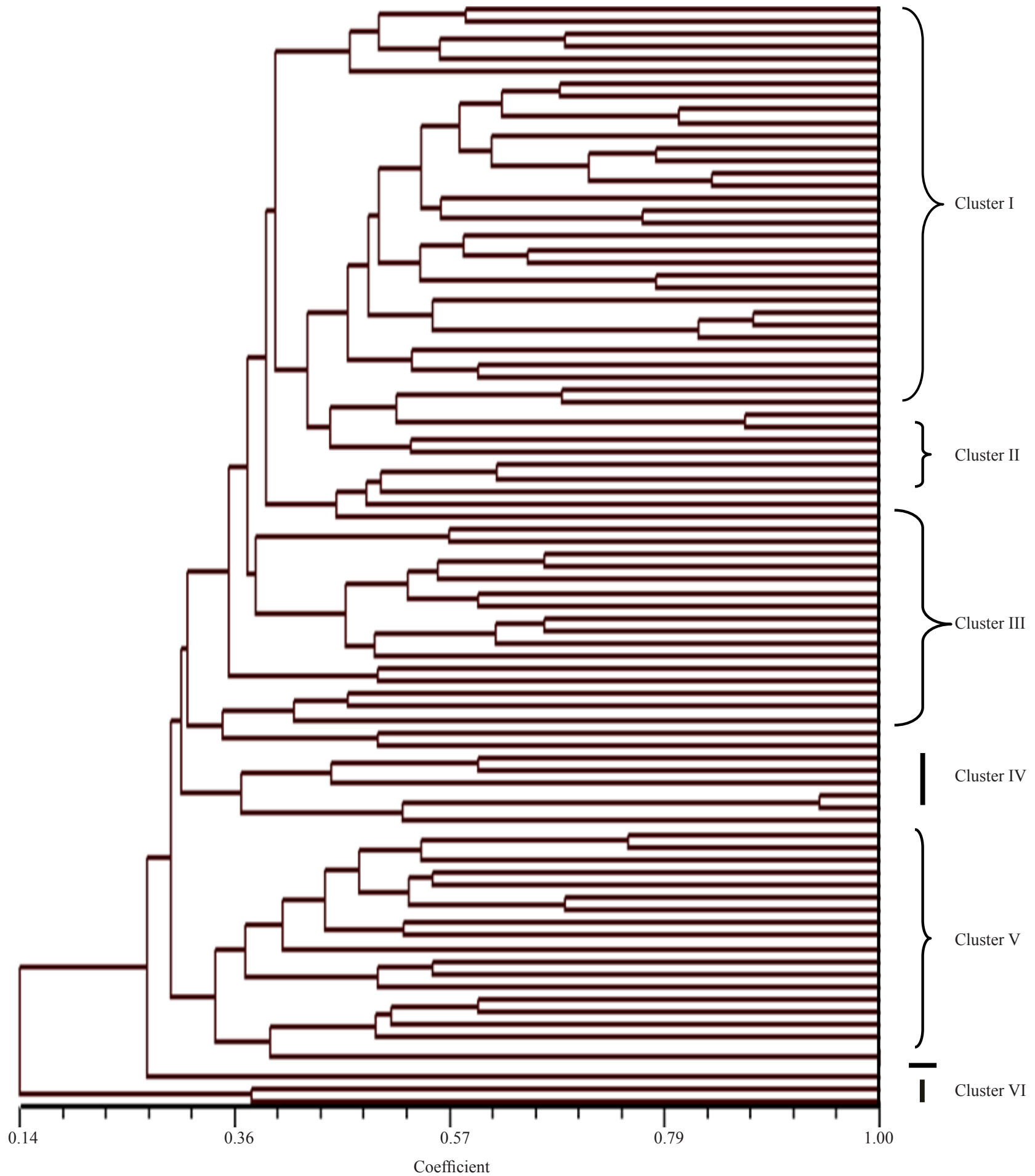

Fig. 2. Jaccard's similarity grouping of 87 chickpea genotypes

range of applications in genetic and breeding of chickpea has been well demonstrated by a number of researchers (Huttel et al., 1999; Winter et al., 2000; Flandez-galvez et al., 2003; Choumane et al., 2000). Microsatellite genotypic data from a number of loci have potential to give unique allelic profiles or DNA fingerprints for establishing genotypes identity (Bharadwaj et al., 2010). A narrow genetic base in chickpea warrants immediate base broadening efforts. Though morphological diversity is generally used by the breeders as a criteria in making

Indian J. Plant Genet. Resour. 28(2): 189-197 (2015) 
Table 3. Clustering of genotypes based on UPGMA analysis of 87 genetic stocks of chickpea using SSR markers

\begin{tabular}{|c|c|c|c|}
\hline S. No. & $\begin{array}{c}\text { Cluster } \\
\text { No. }\end{array}$ & $\begin{array}{c}\text { No. of } \\
\text { genotypes }\end{array}$ & Names of genotypes \\
\hline 1. & I & 36 & $\begin{array}{l}\text { GS1, GS2, GS3, GS4, GS5, GS6, GS7, } \\
\text { GS8, GS10, GS11, GS13, GS14, GS15, } \\
\text { GS16, GS18, GS19, GS20, GS23, GS24, } \\
\text { GS25, GS26, GS27, GS28, GS29, GS30, } \\
\text { GS31, GS41, GS42, GS43, GS44, GS45, } \\
\text { GS49, GS59, GS75, GS85, GS86. }\end{array}$ \\
\hline 2. & II & 5 & GS9, GS12, GS21, GS37, GS40. \\
\hline 3. & III & 13 & $\begin{array}{l}\text { GS17, GS22, GS32, GS36, GS35, GS38, } \\
\text { GS46, GS48, GS55, GS56, GS61, GS68, } \\
\text { GS70. }\end{array}$ \\
\hline 4. & IV & 11 & $\begin{array}{l}\text { GS57, GS69, GS65, GS58, GS60, GS47, } \\
\text { GS52, GS87, GS50, GS51, GS53. }\end{array}$ \\
\hline 5 . & $\mathrm{V}$ & 19 & $\begin{array}{l}\text { GS33, GS34, GS67, GS64, GS66, GS79, } \\
\text { GS80, GS73, GS78, GS74, GS63, GS77, } \\
\text { GS76, GS54, GS81, GS82, GS62, GS83, } \\
\text { GS84. }\end{array}$ \\
\hline 6. & VI & 2 & GS71, GS72. \\
\hline
\end{tabular}

crosses, it is clearly known that the manifestations of ' $\mathrm{G} \times \mathrm{E}$ ' interactions make closely related individuals to appear diverse and thus there are greater chances of these being used in crossing programmes. Knowledge of molecular diversity in the material helps discern this diversity and in identification of parents for crossing programme (Bharadwaj et al., 2010).

For acceleration and optimizing the long process of creating new chickpea cultivars molecular markers are included as analyzing tools. Molecular markers are considered as good candidates for classifying genotypes in different groups and thus assessing genetic distances as well as genetic expected gains. RAPD were earlier used. However owing to greater reliability and repeatability, SSR markers are now being increasingly used for discerning genetic diversity.

Nineteen STMS primer pairs could amplify 1-4 loci per primer pair generating 4.57 allels per locus on an average. Contrary to the fact that chickpea is a self pollinated crop and should generate lower polymorphism. World chickpea germplasm has a narrow genetic base (Nguyen et al., 2004) and lacks the desirable traits needed for ready utilization in varietal improvement programs. A narrow genetic base and sexual incompatibility with other Cicer wild types, which carry the sources for various desirable traits, contribute to the limited progress in the improvement of chickpea yield (Chaudhary et al., 2012). The presence of multiple alleles may have occurred to the fact that there is a very high residual heterozygotic balance conserved due to Desi $\times$ Kabuli introgression that played a major role in chickpea evolution. This may be one of the causes for obtaining multiple bands using SSR markers (Singh et al., 2008). A high degree of molecular polymorphism was exhibited by all the markers studied indicates the markers that have been used for diversity analysis were sound. The PIC ranged from 0.10 to 0.94 and heterozygosity ranged from 0.11 to 0.94 . The Jacards similarity matrix dendogram constructed using the UPGMA method showed that all the clusters were dissimilar and grouped into seven major clusters. A critical examination of these clusters with indicates that the grouping was primarily based on seed size. Cluster VI has SBD 377 and ILC 212. SBD 377, a simple leaf mutant developed at IARI had an ICARDA line in its pedigree PRR1, a derivative from FLIP 90-166, an ICARDA line and thus would have got clustered in proximity with GS72 i.e. ILC 212 . The market collection GS39, a bold seeded kabuli type remained un-grouped as it was very bold in its size and does not represent a released cultivar but market collection obtained under the ISOPOM trial. Cluster two comprised mostly either bold seeded or erect types. Thus plant architecture also played an important role. However, contrary to earlier workers reports, it is clearly noticed that the place where the cultivars were developed did not play a major role in grouping. This may be due to the fact that, the elite breeding lines included in this study obtained from different centers were developed from germplasm either obtained from ICRISAT or ICARDA. In the previous study of Bharadwaj et al. (2011) specific lines of ICARDA, ICRISAT and IARI were used where all the lines from ICARDA and wild species were grouped as a distinct cluster. The breeder's generally use diverse sources selected based on morphological traits and their observation in making crosses. Similar results were obtained by Choudhary et al. (2012).

Results from the present study support the observations of several workers about the potential utility of STMS in characterizing asparagus lines (Huttel et al., 1999; Winter et al., 2000; Flandez-galvez et al., 2003; Choumane et al., 2000). There was reasonably high rate of polymorphism for at least ten markers namely TA194, TA80, TA113, TA117, TA14, TA110, NC6, NC7, CaSTMS15 and NCPGR4 out of 19 STMS markers loci in the present study. This pointed towards the scope for further utilization of these markers for characterization of different cultivars of chickpea. The STMS polymorphism were assayed using a DNA pooling 
strategy, although it is not supposed to do as all the genotypes under study are pure lines (Flandez-Galvez et al., 2003) demonstrated the power and potential of SSR markers for a wide range of applications in genetic and breeding of chickpea. Molecular markers being easily reproducible they have become favourite tools with breeders and biotechnologists to discern the traits as well as to study diversity among cultivars (Satyavathi et al., 2005). However, no correlation could be derived from PIC and allele numbers in this study. Further, Greater gains can be obtained by crossing lines MPJG-2000-108 with SBD 377 for desi improvement and PG 0515 with ILC 212 for kabuli improvement. These genotypes have been identified as most diverse in the present study. Pre-breeding and Base broadening through kabuli $\times$ desi introgression for greater gains is an important activity of the breeders. This increases the diversity obtained in the succeeding generations to carry out further selections as there is enormous amount of variation that is seen in these generations for seed size, seed type and other traits. In the present study greater base broadening can be achieved by using ICC 4516 and ILC 212 as parents in the breeding programme.

This study helped to determine the genetic relationship between elite genetic stock of chickpea based on STMS marker data, and these results greatly contribute to germplasm bank management, conservation programs, and breeding purposes. The occurrence of unique alleles or rare STMS alleles provides an immense opportunity for generation of comprehensive fingerprint database. The present investigation also gives an idea of the interrelationship among the genotypes and highlights the need for helpful supplementation of pedigree data and other morphological data with the database generated by STMS marker to efficiently discover the genetic inter-relationship among the genotypes, fingerprint the varieties for their protection and most importantly select parents for a sound breeding programme.

\section{Acknowledgements}

We acknowledge the funding of ICAR-Indian Agricultural Research Institute and Department of Agricultural Co-operation (DAC), Government of India, and all the partners in the DAC funded project 'Pre-breeding and genetic enhancement in breaking yield barriers in kabuli chickpea and lentil through ICAR-ICARDA collaboration'.

\section{References}

Ahmad F, AI Khan, FS Awan, B Sadia, HA Sadaqat and S Bahadur (2010) Genetic Diversity of Chickpea (Cicer arietinum L.) Germplasm in Pakistan as Revealed by RAPD Analysis. Genet. Mol. Res. 9: 1414-1420.

Bharadwaj C, SK Chauhan, G Rajguru, R Srivastava, CT Satyavathi, S Yadav, AH Rizvi, J Kumar and RK Solanki (2010) Diversity analysis of chickpea (Cicer arietinum) cultivars using STMS markers. Indian J. Agric. Sci. 80: 947-51.

Bharadwaj C, R Srivastava, SK Chauhan, CT Satyavathi, J Kumar, A Faruqui, S Yadav, AH Rizvi and T Kumar (2011) Molecular diversity and phylogeny in geographical collection of chickpea (Cicer sp.) accessions. J. Genet. 90: 94-100.

Choudhary P, SM Khanna, PK Jain, C Bharadwaj, J Kumar, PC Lakhera and R Srinivasan (2012) Genetic structure and diversity analysis of the primary gene pool of chickpea (Cicer arietinum L.) using SSR markers. Genet. Mol. Res. 11: 891-905.

Choumane W, P Winter, F Weig and G Kahl (2000) Conservation and variability of STMS from chickpea within the genus Cicer. Theor. Appl. Genet. 101: 269-278.

Food and Agricultural Organization (2006) http://www.fao.org/ waicent/statistic.asp

Food and Agriculture Organization (2010) Global area, production and productivity of chickpea. http://faostat.fao.org/site/567/ DesktopDefault.aspx [2012 Jun. 12].

Flandez-Galvez H, R Ford, ECK Pang and PWJ Taylor (2003) An intraspecific linkage map of the chickpea (Cicer arietinum L.) genome based on the sequence microsatellite site and resistance gene analog markers. Theor. Appl. Genet. 106: 1447-1456.

Goldstein DB and DD Pollock (1997) Launching microsatellites: a review of mutation processes and methods of phylogenetic inference. J. Heredity 88: 335-342.

Hüttel B, P Winter, K Weising and G Kahl (1999) Sequence Tagged Microsatellite markers for chickpea (Cicer arietinum L.) Genome 42: 210-217.

Jarne P and PJL Lagoda (1996) Microsatellites, from molecules to populations and back. Ecol. Evol. 11: 424-430.

Kumar T, C Bharadwaj, C Tara Satyavathi and PK Jain (2013) A high throughput, improved rapid and reliable genomic DNA extraction protocol from chickpea (Cicer arietinum L.) Vegetos 26(2): 185-190.

Lynch M and Walsh JB (1998) Genetics and Analysis of Quantitative Traits. Sinauer Assocs., Inc., Sunderland, MA.

Nawroz ART and FHK Hero (2011) Determination of genetic relationship among some varieties of Chickpea (Cicer arietinum L.) in Sulaimani by RAPD and ISSR Markers. Jordan J. Biomed. Sci. 4: 77-86.

Nguyen TT, PWJ Taylor, RJ Redden and R Ford (2004) Genetic diversity estimates in Cicer using AFLP analysis. Plant Breed. 123: 173-179.

Powell W, GC Marchy and J Provan (1996) Polymorphism revealed by simple sequence repeats. Trends Plant Sci. 1: 215-222. 
Singh R, P Sharma, RK Varshney, SK Sharma, NK Singh (2008) Chickpea improvement: role of wild species and genetic markers. Biotechnol Genet. Eng. Rev. 25: 267-313.

Singh PK, H Sharma, N Srivastava, SS Bhagyawant (2014) Analysis of Genetic Diversity among Wild and Cultivated Chickpea Genotypes Employing ISSR and RAPD Markers. American J. Plant Sci. 5: 676-682.

Satyavathi CT, KV Bhat, C Bharadwaj, SP Tiwari, and V Chaudhary (2006) AFLP analysis of genetic diversity in Indian soybean [Glycine $\max (\mathrm{L}$.) Merrill] varieties. Genet. Res. Crop Evol. 53: 1069-1079.

Upadhyaya HD, M Thudi, N Dronavalli, N Gujaria, S Singh, S Sharma and RK Varshney (2011) Genomic tools and germplasm diversity for chickpea improvement. Plant Genet. Resour. 9: 45-58.

Varshney RK, Song chi and RK Saxena (2013) Draft genome sequence of chickpea (Cicer arietinum) provides a resource for trait improvement. Nature Biotechnol. 31: 240-246.

Winter P, AM Benko-Iseppon, B Huttel, M Ratnaparkhe, G Tullu and G Sonnante (2000) A linkage map of the chickpea (Cicer arietinum $\mathrm{L}$ ) genome based on recombinant inbred lines from a $C$. arietinum $\times$ C. reticulatum cross: localization of resistant genes for fusarium wilt races 4 and 5. Theor. Appl. Genet. 101: 1155-1163. 